\title{
Image Segmentation Methods for Detecting Blood Vessels in Angiography
}

\author{
Albert C. S. Chung \\ Lo Kwee-Seong Medical Image Analysis Laboratory, \\ Department of Computer Science and Engineering, \\ The Hong Kong University of Science and Technology, Clear Water Bay, Hong Kong \\ Email: achung@cse.ust.hk
}

\begin{abstract}
Computer-assisted detection and segmentation of blood vessels in angiography are crucial for endovascular treatments and embolization. In this article, I give an overview of the image segmentation methods using the features developed recently at our laboratory. Our current research directions are also highlighted.
\end{abstract}

Keywords-Segmentation of Blood Vessels, Feature Detection, Statistical Segmentation, Active Contour Model, Angiography

\section{INTRODUCTION AND MOTIVATION}

Segmentation of blood vessels is one of the essential medical computing tools for clinical assessment of vascular diseases. It is a process of partitioning an angiogram into nonoverlapping vascular and background regions. Based on the partitioning results, surfaces of vasculatures can be extracted, modeled, manipulated, measured and visualized. These are very useful and play important roles for the endovascular treatments of vascular diseases. Vascular diseases are one of the major sources of morbidity and mortality worldwide. Therefore, developing reliable and robust image segmentation methods for angiography has been a priority in our group and other research groups.

It is challenging to perform image segmentation in angiography. For example, blood vessels can contain low or complex flow. This can lead to low signal-to-noise (SNR) ratio in the angiograms. The conventional segmentation methods based on image intensity alone may then fail when there is a significant signal drop in the vascular region. Furthermore, the intensity inhomogeneity violates the intensity piecewise constant assumption in the segmentation process. Finally, the intensity contrast between vessel and background regions, or inside vessel regions can vary from region to region. Therefore, the local intensity statistics in the vessel and background regions may not be reliable, or the intensity gradient magnitude may not be large enough on the vessel boundary for the conventional image segmentation methods. Reviews on this topic can be found in [1], [2]

This paper reports the image segmentation methods recently developed at Lo Kwee-Seong Medical Image Analysis Laboratory, The Hong Kong University of Science and Technology for detecting blood vessels in angiography. In Section II, the features for detecting blood vessels are discussed and then frameworks for delineating the vasculatures are presented (see Section III). Finally, the current research directions at our laboratory using the image segmentation methods are discussed in Section IV.

\section{FEATURES FOR DETECTING BLOOD VeSSELS}

Detecting blood vessels in angiography is a core component in segmentation of vasculatures. When analyzing angiograms using the computer-assisted techniques, it is common to detect the blood vessel boundary based on the magnitude of image gradient. However, the gradient magnitude in the angiograms may not provide sufficient information for locating blood vessel boundary and performing reliable vascular segmentation. To improve image segmentation quality, rather than using the gradient magnitude alone, it is our focus to develop new image features for blood vessel segmentation in angiography using additional information about local blood flow coherence, local iso-intensity structural orientation and weighted local variances of image intensity.

\section{A. Local Phase Coherence}

Phase contrast magnetic resonance angiography (PC-MRA) provides the speed-dependent images, in which the background and vascular regions are given high intensity contrast. In addition, PC-MRA gives the measured $x, y$ and $z$ velocity components of the flow vectors on a voxel-by-voxel basis. This measured information is presented in the form of phase images along the three principle axes, $x, y$ and $z$. Phase images give directional information about the local blood flow velocity field and blood motion in the brain. Using this information, we have developed a measure using local phase coherence (LPC) to quantify locally coherent flow patterns and random flow patterns [3].

On the phase images, LPC measures the local flow coherence based on the sum of dot products of all adjacent flow vector pairs inside a pre-defined image window. It is effective for capturing the spatial relationship between adjacent flow vectors in the image window, and thus distinguishing the coherent and random flow patterns. Similar to the image texture analysis, blood vessel boundary is then defined as the discontinuity between locally coherent and random flow patterns. The use of LPC in segmenting blood vessels and related research work has been demonstrated by our group and other groups [4], [5], [6], [7], [8], [9]. 


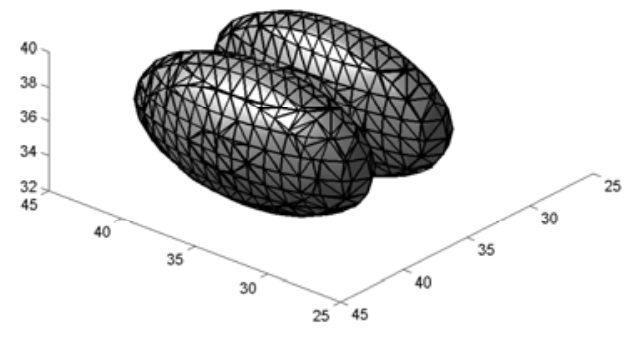

Fig. 1. 3D surface of the filters.

\section{B. Local Iso-Intensity Structural Orientation}

Local image structure and its orientation can be estimated using the orientation tensor, which combines the outputs from a number of directional polar separable quadrature filters [10]. The quadrature filter is a complex valued filter in the spatial domain. It can be constructed in the Fourier domain and makes the implementation more efficient. The real part of the filter can be treated as a line filter because of its symmetric filter response and the imaginary part can be viewed as an edge filter because of the asymmetric response. The filter can give good responses to these local structures (i.e., lines and edges) even though there is smooth intensity non-uniformity in the images.

We believe that the local structures and their estimated orientations are useful prior knowledge for the segmentation of blood vessels [11]. This is so because, inside blood vessels in the angiography, the local iso-intensity structures should exist if the vessel surfaces are coherent. Since it is structureless in the background regions due to the random noise, the estimated orientations can only be applied to vascular regions. To improve the quality of the binary segmentation of angiograms, we have developed a method to exploit this local structural coherence such that the piecewise homogeneous assumption of image objects (i.e., blood vessels) can be relaxed in image segmentation. Therefore, the blood vessel boundary is defined along the coherent local structures. This feature has been tested on synthetic and clinical PC-MRA images.

\section{Weighted Local Variances}

Low-contrast and thin vascular regions in the angiograms are not easy to handle in the segmentation process. To deal with this problem, a number of approaches have been proposed. In the low-contrast regions, rather than using the unreliable intensity gradient alone, contours can also be evolved based on the shape prior. For example, there are methods which use tubular template matching for vessel detection, [12], [13], [14].

Instead of employing shape priors, the CURVE algorithm [15] proposed by Lorigo et al. uses the smaller principal curvature to keep the curve evolving in tubular shape along the orientation of blood vessels. Also, Yan and Kassim proposed the use of "capillary force" in the geodesic active contours
[16]. This force is capable of pulling the evolving curves into low-contrast and thin vascular regions. Vasilevskiy and Siddiqi proposed the "Flux Maximizing Geometric Flows" [17], in which the flux is computed in a multi-scale fashion and the maximum response is chosen to be the value of flux.

We have developed a new blood vessel boundary detection scheme. This new scheme is independent of image intensity contrast for segmentation of low-contrast and thin vessels. The new feature is based on the weighted local variance (WLV). WLV estimates the local intensity variance weighted by the first derivative of a Gaussian function, which is rotated to align with a given orientation. The first derivative of a 3D Gaussian function is shown in Figure 1. At each voxel, WLVs can be estimated along different discrete orientations.

WLVs are useful in extracting blood vessel boundary information consisting of both boundary orientation and boundary magnitude. The boundary orientation can be estimated in a continuous fashion using the relationship between WLVs obtained along different discrete orientations. Boundary magnitude estimated using the WLVs depends on the clarity of the boundary. The advantage is that the estimated magnitude does not depend on image intensity contrast. This feature can help prevent contours from being trapped inside highcontrast and low-contrast transition regions. Thus, the evolving contours can continue to propagate from high-contrast regions to low-contrast regions. This feature has been tested in digital subtraction angiography (DSA), PC-MRA and 3D rotational angiography (3DRA). As an example, Figure 2 shows the results on a PC-MRA data set. Figures 2(a) and 2(b) show an image slice and its boundary magnitude response based on WLV respectively. Figure 2(c) shows the maximum intensity projection of the data set. 3D extracted boundary surfaces are shown in Figure 2(d).

\section{Segmentation Algorithms using Image FEATURES}

\section{A. Statistical Segmentation}

Image segmentation problem can be formulated in the Bayesian framework. This is a probabilistic framework for estimating the posterior probability based on the product of observation model and prior model. The observation model embodies the knowledge of image formation and noise properties. The prior model represents the prior beliefs about the image. Our group has proposed new observation models and prior models based on the aforementioned features [18], [3], [11], as discussed in Section II.

We exploited the physics of PC-MRA image formation in the formulation of the observation model using the finite statistical mixture models. The overall probabilistic density function of a PC-MRA speed-dependent image can be described as either a Maxwell-uniform (MU) or Maxwell-Gaussian-uniform (MGU) mixture model. Experimental results show that the proposed statistical mixture models can provide a better modeling of the statistical properties of the underlying background and vascular signals. This is very useful in the segmentation process. Works have been proposed by our group and other 


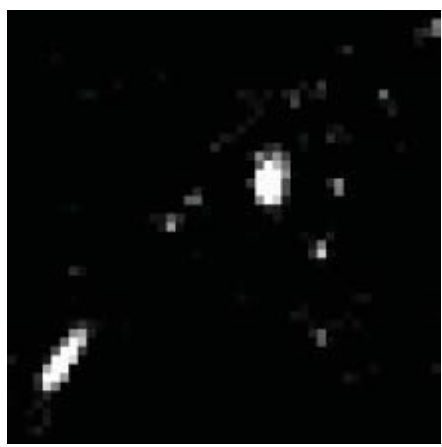

(a) A slice of the data set

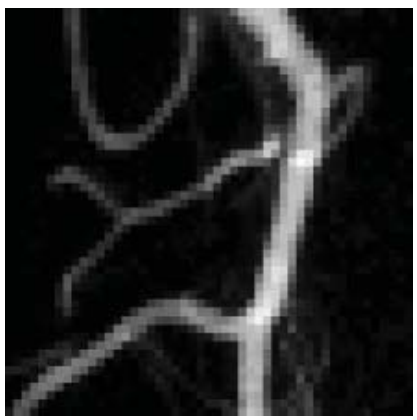

(c) MIP

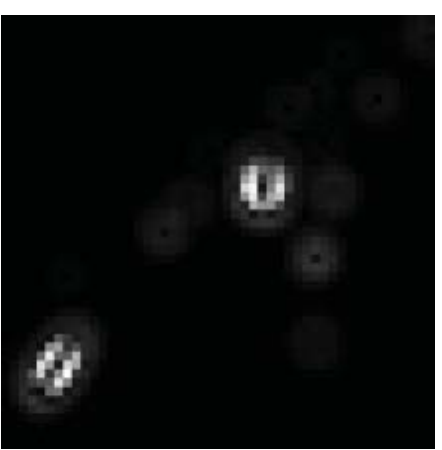

(b) WLV response magnitude

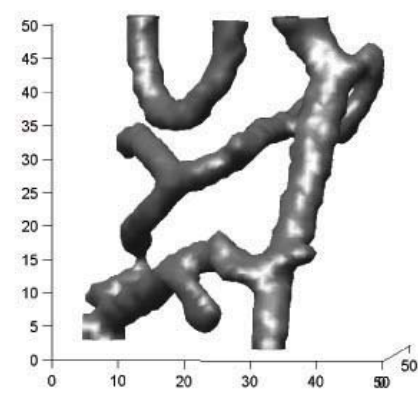

(d) 3D surfaces

Fig. 2. Results on a PC-MRA data set. (a) A slice of the data set. (b) The boundary magnitude response of WLV. (c) Maximum Intensity Projection (MIP). (d) 3D surfaces of the segmented vessels.

groups [19], [20], [21], [22] using similar research principles based on different statistical mixture models.

The prior model using the local phase coherence was explored to encourage flow coherence in the segmented vascular regions. This gives better delineation of the blood vessel boundary between vascular regions and background regions [3]. Along the same research line, another new prior model using the local iso-intensity structural orientations was proposed. The model ensures the local structural coherence of the vascular surface and constrains the binary segmentation within the Bayesian framework [11]. It is experimentally shown that the new observation models and prior models can further improve the robustness of the segmentation methods when $\mathrm{SNR}$ is low in the images.

\section{B. Segmentation using Active Contour Model}

Apart from using the statistics of image intensity, image segmentation methods using the active contour models have been an active research area. Given the estimated boundary information using the weighted local variance, we have formulated an active contour model for vessel boundary delineation. The model performs segmentation by minimizing the weighted angular discrepancy between contour and boundary orientation. The weights are determined by the boundary magnitude. The level set method is used for the ease of implementation and handling of topological changes.

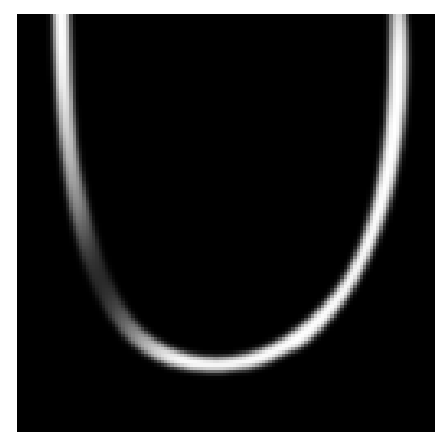

(a) Vessel image

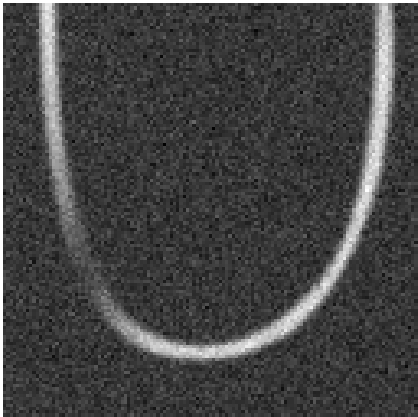

(c) Image with noise

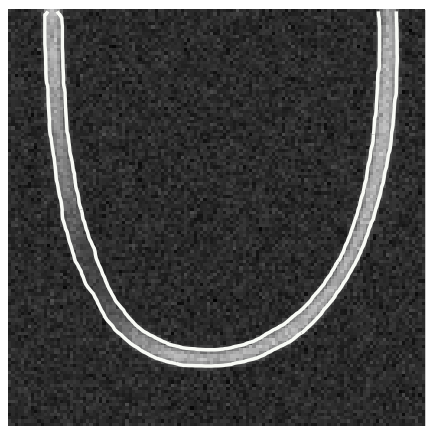

(e) Final contour

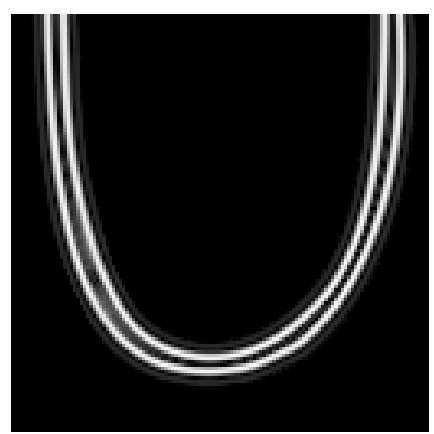

(b) WLV response magnitude

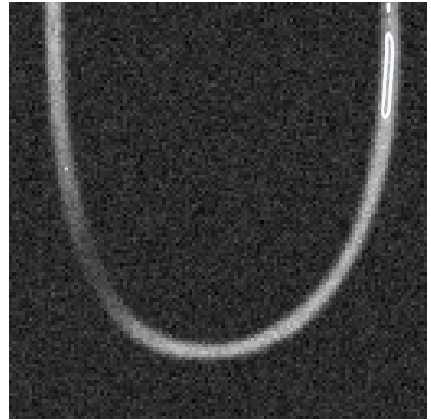

(d) Initial contours

Fig. 3. Results on a synthetic image. (a) The synthetic image slice containing a U-shape tube. (b) The boundary magnitude response of WLV. (c) Noise corrupted synthetic image. (d) Initial contours (top right portion of the $U$ shape tube). (e) Final contour.

For a further illustration, a synthetic image was generated. The image is shown in Figure 3(a). It contains a U-shape tube in the image. The estimated boundary magnitude is displayed in Figure 3(b). The synthetic image was then corrupted by a Gaussian noise (see Figure 3(c)). The initial contours and final contour are drawn in Figures 3(d) and 3(e) respectively. It is observed that even though the intensity values in the Ushape tube have high-contrast and low-contrast transitions, the contour can still be able to propagate through these regions.

\section{CONCLUSiOn AND RESEARCH DiRECTIONS}

To conclude, while it is common to use gradient magnitude for detecting the blood vessel boundary and performing image segmentation in angiography, there are additional features 
that can be used for more robust and reliable blood vessel segmentation in angiography. We have demonstrated the incorporation of additional blood flow information provided by the imaging devices and the use of physics of the image formation in the statistical segmentation process such that the uncertainty about image intensity can be better modeled. Moreover, the coherence of local iso-intensity structures in the vascular regions can impose an additional structural smoothness constraint in image segmentation. Finally, the problem of segmenting low-contrast vascular regions can be handled by using intensity independent features, such as weighted local variance, and an active contour model based on weighted angular discrepancy minimization. The immediate next step is to develop a unified image segmentation framework for effectively combining different image features.

In general, using the image segmentation methods presented in this paper, the segmented brain vessels in the angiography should be used and it is demonstrated to give useful information for the endovascular treatments. We will discuss two current research directions using the image segmentation methods developed at our laboratory.

The first work is related to the identification and quantitative analysis of vascular abnormalities. An augment vessel [23] refers to a computer-generated vessel for estimating a portion of post-treatment vessel lumens under conditions that either (1) a stent successfully restores the width of a stenotic lumen which is comparable to the widths of normal lumen segments that are proximal and distal to the coarctation, (2) an aneurysmal sac is completely packed with GDC, or (3) an aneurysmal lumen is occluded perfectly by stent grafts [24]. Those conditions are regarded as clinically ideal, since the post-treatment vessel lumens approximated are very similar to normal lumens.

We are developing a new unified framework which uses the recently developed augmented vessel method to identify and quantify a variety of vascular abnormalities, e.g. stenotic atherosclerotic plaque, saccular and fusiform aneurysmal lumens, from segmented vasculatures. Different from other methods, our method models the opposite of the abnormalities to locate the lesion lumens in an indirect fashion. The advantage is that the normal vessel models (i.e., augmented vessels) are easier to manipulate as compared with the model of the complex shaped disease lumens.

For example, Figure 4 shows how the augmented vessels can be applied to the detachment of aneurysmal lumens from the segmented vasculatures. Figure 4(a) shows our result on a 3DRA data set that contains a wide-neck aneurysm at the bifurcation of ACA and ACoA. Figure 4(b) illustrates the centerlines of the augmented vessels estimated. Figures 4(c) and 4(d) show the manually delineated approximation of posttreatment lumens under the condition of a perfect embolization and the manually drawn cardinal splines. From Figure 4, high similarity between the augmented vessel centerlines and the trajectories of the approximated post-treatment lumens is noticed. Figure 5 shows an encouraging result on a 3DRA data set that contains a coarctation of the MCA.

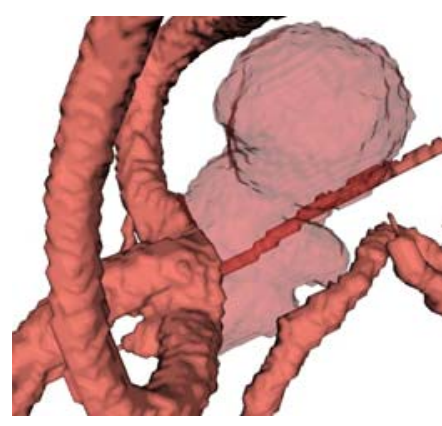

(a) Our method

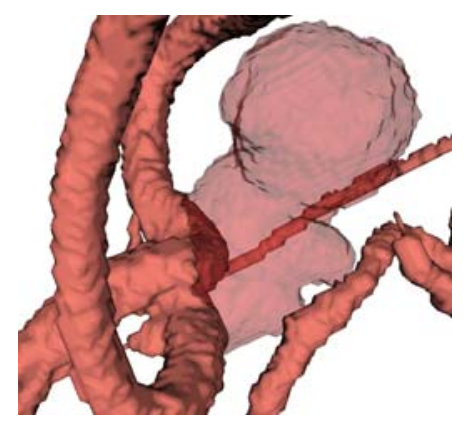

(c) Manual delineation

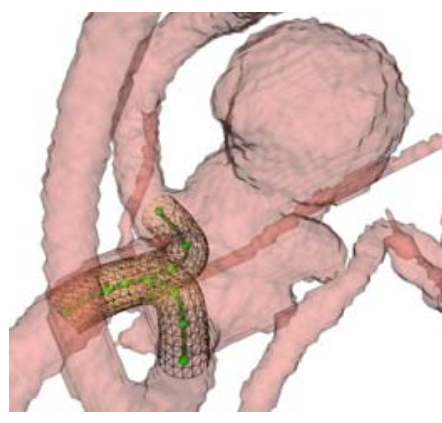

(b) Centerlines of the augmented vessels estimated

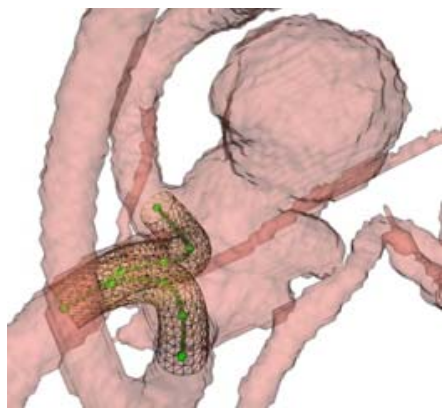

(d) Manually drawn splines
Fig. 4. (a) Our result on a 3DRA data set that contains a wide-neck aneurysm at the bifurcation of ACA and ACOA. (b) The estimated centerlines of the augmented vessels. (c) Manually delineated approximation of post-treatment lumens under the condition of a perfect embolization. (d) Manually drawn cardinal splines.

The second research direction is the enhanced visualization of the angiograms using vessel boundary information. For diagnosis of vascular diseases and effective endovascular planning, direct volume rendering (DVR) is an effective and widely used technique for vascular image volume visualization. In [25], we proposed a framework that uses the Hessian-based image enhancement methods to achieve better DVR quality. Figure 6 shows the results on a 3DRA data set. Figure 6(a) shows a maximum intensity projection (MIP) of the data set. Figures 6(b) and 6(c) show the results based on the conventional one-dimensional DVR transfer function. Finally, results obtained using the new multi-dimensional transfer function are illustrated in Figure 6(d). We believe that with better delineation of blood vessel boundary based on accurate image segmentation methods the DVR quality can be further improved.

\section{ACKNOWLEDGMENTS}

I would like to thank Max Law, Wilbur Wong and Vincent Yuan at our laboratory, and Ming-Yuen Chan and Huamin $\mathrm{Qu}$ at our department for their contributions in the projects discussed in this paper; and thank Alison Noble at Wolfson Medical Vision Laboratory and Paul Summers at The Nuffield Department of Surgery, The University of Oxford, U.K. for their contributions in the development of the local phase coherence feature. I would also like to thank Simon Yu at Prince of Wales Hospital, Hong Kong for his contribution 


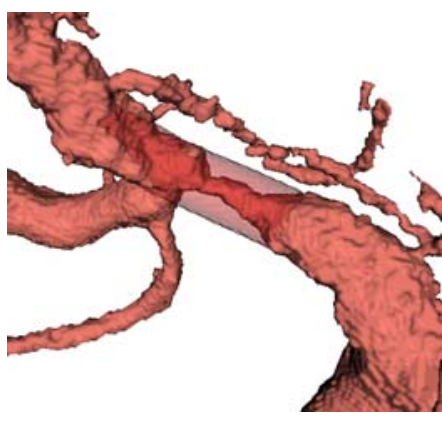

(a) Our method

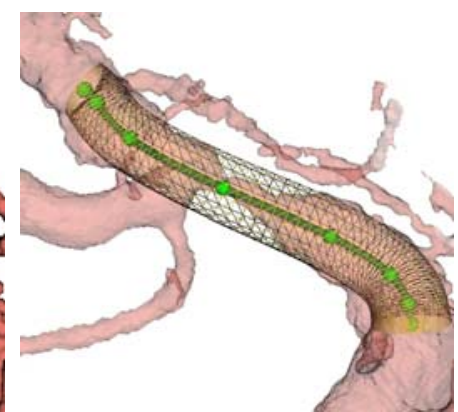

(b) Centerlines of the augmented vessels estimated
Fig. 5. (a) Our result on a 3DRA data set that contains a coarctation of the MCA. The estimated atherosclerotic plaque volumes are presented by semitransparent surface. (b) The estimated centerlines of the augmented vessels.

in developing the augmented vessels and for providing clinical data sets. This work is partially supported by the $\mathrm{K}$ S Lo foundation, the Hong Kong Research Grants Council under Grants HKSUT6209/02E, DAG01/02.EG04, 612305, and the Sino Software Research Institute (SSRI) under Grant SSRI01/02.EG22.

\section{REFERENCES}

[1] J. S. Suri, K. Liu, L. Reden, and S. Laxminarayan, "A Reivew on MR Vascular Image Processing Algorithms: Acquisition and Prefiltering: Part I," IEEE Trans. Information Technology in Biomedicine, vol. 6, no. 4, pp. 324-337, 2002.

[2] — " "A Reivew on MR Vascular Image Processing Algorithms: Skeleton Versus Nonskeleton Approaches: Part II," IEEE Trans. Information Technology in Biomedicine, vol. 6, no. 4, pp. 338-350, 2002.

[3] A. C. S. Chung, J. A. Noble, and P. Summers, "Vascular Segmentation of Phase Contrast Magnetic Resonance Angiograms Based on Statistical Mixture Modeling and Local Phase Coherence," IEEE Trans. Med. Imag., vol. 23, no. 12, pp. 1490-1507, 2004.

[4] A. L. N. Wong, H. Liu, and P. Shi, "Segmentation of myocardium using velocity field constrained front propagation," in The Sixth IEEE Workshop on Applications of Computer Vision (WACS'02), 2002, pp. 1-6.

[5] J. H. Hipwell, G. P. Penney, T. C. Cox, J. V. Byrne, and D. J. Hawkes, " $2 \mathrm{~d}-3 \mathrm{~d}$ intensity based registration of dsa and mra - a comparison of similarity measure," in International Conference on Medical Image Computing and Computer-Assisted Intervention (MICCAI'02), vol. LNCS 2489, 2002, pp. 501-508.

[6] H. Liu, A. L. N. Wong, and P. Shi, "Cardiac motion and material properties analysis using data confidence weighted extended kalman filter framework," in IEEE International Conference on Acoustics, Speech, and Signal Processing (ICASSP'03), vol. III, 2003, pp. 465-468.

[7] J. H. Hipwell, G. P. Penney, R. A. McLaughlin, K. Rhode, P. Summers, T. C. Cox, J. V. Byrne, J. A. Noble, and D. J. Hawkes, "Intensity-Based 2-D-3-D Registration of Cerebral Angiograms,' IEEE Trans. Med. Imag., vol. 22, no. 11, pp. 1417-1426, 2003.

[8] P. Shi and H. Liu, "Stochastic finite element framework for simultaneous estimation of cardiac kinematic functions and material parameters," Medical Image Analysis, vol. 7, no. 4, pp. 445-464, 2003.

[9] P. Armitage, C. Behrenbruch, M. Brady, and N. Moore, "Extracting and visualizing physiological parameters using dynamic contrast-enhanced magnetic resonance imaging of the breast," Medical Image Analysis, vol. 9, no. 4, pp. 315-329, 2005.

[10] H. Knutsson, "Representing Local Structure Using Tensors," in The 6th Scandinavian Conference on Image Analysis (SCIA'89), 1989, pp. 244251.

[11] W. C. K. Wong and A. C. S. Chung, "Bayesian Image Segmentation Using Local Iso-intensity Structural Orientation," IEEE Trans. Image Processing, vol. 14, no. 19, pp. 1512-1523, 2005.

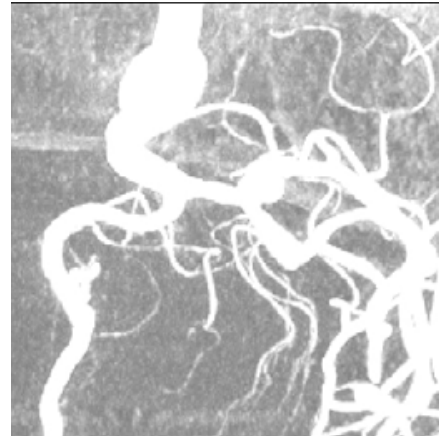

(a) MIP of a 3DRA data set

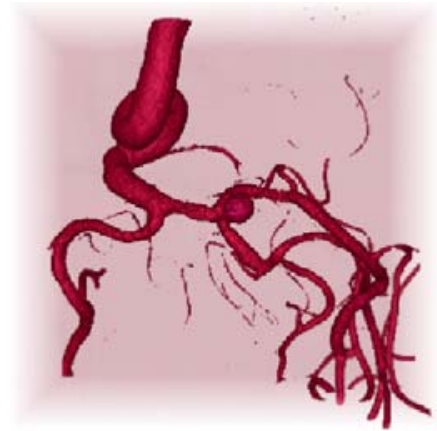

(b) Results using 1DTF

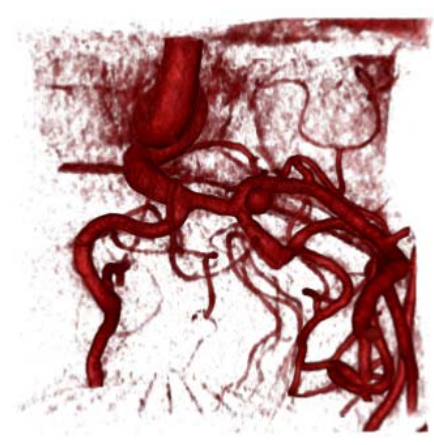

(c) Results using 1DTF

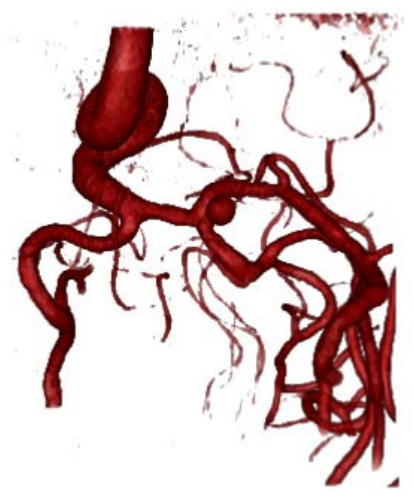

(d) Results using MDTF
Fig. 6. Results on a 3D-RA data set. (a) Maximum Intensity Projection (MIP); (b) and (c) Results using $1 D$ transfer function (1DTF); (d) Results using our multi-dimensional transfer function (MDTF).

[12] E. Bullitt, S. Aylward, A. Liu, J. Stone, S. K. Mukherji, C. Coffey, G. Gerig, and S. M. Pizer, "3d graph description of the intracerebral vasculature from segmented mra and tests of accuracy by comparison with X-ray angiograms," in The Information Processing in Medical Imaging (IPMI'99), vol. LNCS 1613, 1999, pp. 308-321.

[13] K. Krissian, G. Malandain, N. Ayache, R. Vaillant, and Y. Trousset, "Model-based multiscale detection of 3d vessels," in IEEE International Conference on Computer Vision and Pattern Recognition (CVPR'98), vol. I, 1998, pp. 722-727.

[14] D. Nain, A. Yezzi, and G. Turk, "Vessel Segmentation using a Shape Driven Flow," in International Conference on Medical Image Computing and Computer-Assisted Intervention (MICCAI'04), vol. LNCS 3216, 2004, pp. 51-59.

[15] L. M. Lorigo, O. D. Faugeras, W. E. L. Grimson, R. Keriven, R. Kikinis, A. Nabavi, and C.-F. Westin, "CURVES: Curve Evolution for Vessel Segmentation," Medical Image Analysis, vol. 5, no. 3, pp. 195-206, 2001.

[16] P. Yan and A. A. Kassim, "MRA Image Segmentation with Capillary Active Contour," in International Conference on Medical Image Computing and Computer-Assisted Intervention (MICCAI'05), vol. LNCS 3749, 2005, pp. 51-58.

[17] A. Vasilevskiy and K. Siddiqi, "Flux Maximizing Geometric Flows," IEEE Trans. Pattern Anal. Machine Intell., vol. 24, pp. 1565-1578, 2002.

[18] A. C. S. Chung, J. A. Noble, and P. Summers, "Fusing Magnitude and Phase Information for Vascular Segmentation in Phase Contrast MR Angiograms," Medical Image Analysis, vol. 6, pp. 109-128, 2002.

[19] R. Gan, W. C. K. Wong, and A. C. S. Chung, "Statistical Cerebrovascular Segmentation in Three-Dimensional Rotational Angiography Based on Maximum Intensity Projections," Medical Physics, vol. 32, no. 9, pp. 3017-3028, 2005.

[20] M. S. Hassouna, A. A. Farag, S. Hushek, and T. Moriarty, "Statisticalbased approach for extracting 3d blood vessels from tof-mra data," in International Conference on Medical Image Computing and ComputerAssisted Intervention (MICCAI'03), vol. LNCS 2878, 2003, pp. 680-687.

[21] F. Jia, S. Wang, L. Liu, and H. Li, "Preprocessing based statistical seg- 
mentation of mra dataset," in ACM SIGGRAPH international conference on Virtual Reality continuum and its applications in industry, 2004, pp. 306-308.

[22] M. S. Hassouna, A. Farag, S. Hushek, and T. Moriarty, "Cerebrovascular segmentation from TOF using stochastic models," Medical Image Analysis, vol. 10, no. 1, pp. 2-18, 2006.

[23] W. C. K. Wong and A. C. S. Chung, "Augmented Vessels for Quantitative Analysis of Vascular Abnormalities and Endovascular Treatment Planning," IEEE Trans. Med. Imag., To appear.

[24] M. Egmont-Petersen, D. de Ridder, and H. Handels, Vascular and interventional radiology: principles and practice, 2002.

[25] M.-Y. Chan, Y. Wu, H. Qu, A. C. S. Chung, and W. C. K. Wong, "Mipguided vascular image visualization with multi-dimensional transfer function," in Computer Graphics International 2006, To appear. 